\title{
Association between Tumor Characteristics and Bone Mineral Density in Postmenopausal Breast Cancer Patients
}

\author{
Soley Bayraktar, MD, MBA, ${ }^{*}$ Tiffany Avery, MD,${ }^{\dagger}$ Kadri Altundag, MD, ${ }^{\S}$ \\ Kristine Broglio, MS, and Banu K. Arun, $\mathrm{MD}^{\dagger}$ \\ *Division of Cancer Medicine, The University of Texas MD Anderson Cancer Center, Houston, Texas; \\ ${ }^{\dagger}$ Department of Breast Medical Oncology, The University of Texas MD Anderson Cancer Center, \\ Houston, Texas; ${ }^{\star}$ Department of Biostatistics, The University of Texas MD Anderson Cancer Center, \\ Houston, Texas; and ${ }^{\S}$ Department of Medical Oncology, Hacettepe University School of Medicine, \\ Ankara, Turkey
}

Abstract: The aim of this study was to evaluate the association of bone mineral density (BMD) at the time of diagnosis with clinical-pathologic findings in patients with operable postmenopausal breast cancer. One hundred and fifty-eight postmenopausal women who had a baseline lumbar and hip BMD measurement were included in the analysis. Patients were divided into two groups based on the median BMD. $p \leq 0.002$ was considered to be statistically significant. Hormone replacement therapy (HRT) use longer than 5 years was associated with increased lumbar BMD compared with patients who used HRT less than 5 years $(p=0.002)$. Patients with higher BMD tended to have low grade disease, no lympho-vascular invasion, progesterone receptor-positive tumors, and low Ki-67 levels $(p<0.05)$. Higher baseline BMD in postmenopausal patients with breast cancer is associated with favorable prognostic features.

Key Words: bone mineral density, breast cancer, postmenopausal, prognostic factors

A part from family history and genetic predisposition, there is substantial evidence that lifetime exposure to endogenous and exogenous estrogens plays a major role in developing breast cancer in postmenopausal women (1-3). However, the use of serum hormone levels clinically and in risk prediction models has been difficult due to variations in measurement between laboratories and serum fluctuations (4). Thus, surrogate markers of endogenous estrogen levels, such as bone mineral density (BMD), have been explored as an alternative means to measure serum endogenous estrogen levels $(5,6)$.

As the proposal of BMD as a biomarker for cumulative estrogen exposure in a woman's lifetime, the association between increased BMD and risk of breast cancer

Address correspondence and reprint requests to: Soley Bayraktar, MD, MBA, Department of Breast Medical Oncology, The University of Texas MD Anderson Cancer Center, 1515 Holcombe Boulevard, Unit 1354, Houston, TX 77030-4009, USA, or e-mail: soley.bayraktar@gmail.com.

Disclaimers: The manuscript has never been published and is not under consideration for publication elsewhere. All authors have no conflict of interest to declare.

DOI: $10.1111 /$ tbj.12141

(C) 2013 Wiley Periodicals, Inc., 1075-122X/13

The Breast Journal, Volume 19 Number 4, 2013 431-434 in postmenopausal women has been established by a number of studies (7-14). Little is known, however, about the prognostic characteristics of the tumors that develop in postmenopausal women with higher endogenous levels of estrogen. Therefore, we conducted this retrospective analysis to analyze the association between the favorable tumor characteristics and higher BMD in postmenopausal women with breast cancer.

\section{MATERIALS AND METHODS}

\section{Patient Population and Data Collection}

The Breast Cancer Management System database of the University of Texas MD Anderson Cancer Center (MDACC) identified 158 postmenopausal women who were diagnosed with operable breast cancer, and for whom BMD was measured at the time of diagnosis, between 1997 and 2011. This study was approved by the MDACC Institutional Review Board.

Because the composition and metabolism of bone are not uniform throughout the skeleton, measurements of 
BMD (expressed in $\mathrm{g} / \mathrm{cm}^{2}$ ) were made at more than one skeletal site. Lumbar spinal and total hip BMD were measured by dual-energy X-ray absorptiometry (DXA). Osteoporosis and low BMD were defined by the World Health Organization (WHO) criteria based on DXAderived T-scores for total hip.

Pathologic Assessment and Mutation Analysis Initial clinical and pathologic stage of all patients were based on the sixth edition of the American Joint Committee on Cancer staging criteria (15). Nuclear staining $\geq 1 \%$ of estrogen receptor (ER) or progesterone receptor (PR) was considered strongly positive on immunohistochemistry (IHC). HER2 positivity was defined as $3+$ receptor overexpression on IHC staining and/or gene amplification by fluorescence in situ hybridization (HER-2/CEP $17 \geq 2$ were considered as amplified). Nuclear staining $\geq 17 \%$ of Ki67 was considered significant on IHC.

Statistical Analysis and Outcome Measures Patient characteristics were tabulated and the Wilcoxon signedrank test or the Kruskal-Wallis test was used to determine whether the distribution of BMD was associated with patient characteristics. Due to the number of statistical tests performed, only $\mathrm{p} \leq 0.002$ were considered to be statistically significant; all tests were twosided. Statistical analysis was carried out using SAS 9.1.3 (SAS Institute, Cary, NC) and S-Plus 8.0 (Insightful Corporation, Seattle, WA).

\section{RESULTS}

Hip BMD was significantly lower in patients older than 60 years of age at the time of diagnosis compared with patients younger than 60 years of age $(\mathrm{p}=0.002)$. Hormone replacement therapy (HRT) use longer than 5 years was associated with increased lumbar BMD compared with patients who used HRT $<5$ years. Additionally, patients with higher BMD tended to have low grade disease, no lympho-vascular invasion (LVI), PR-positive tumors and low Ki-67 levels $(\mathrm{p}<0.05)$. Likewise, patients with low BMD tended to have larger tumors and positive lymph nodes at the time of diagnosis (Table 1).

In the subgroup analyses of patients 60 years of age or less, no patient or disease characteristics were found to be significantly associated with either lumbar $\mathrm{BMD}$ or hip BMD. Likewise, no significant associations between the clinical characteristics and BMD were observed among the subgroup of patients older than 60 years of age (data not shown).

After adjustment for age, HRT use more than 5 years was associated with higher baseline lumbar and hip BMD. Also, patients with higher lumbar BMD had a significantly higher rate of tumors with low nuclear grade, PR-positive status, low Ki-67 levels, and negative LVI. And patients with higher hip BMD tended to have negative lymph nodes at the time of initial diagnosis (Table 2).

\section{DISCUSSION}

In this study, we examined patient and tumor characteristics in relation to BMD in postmenopausal patients with breast cancer. Our objective was to determine whether there were any associations between higher endogenous estrogen levels, for which BMD served as a surrogate marker, and favorable breast tumor characteristics. Our data indicate that tumor characteristics are not significantly associated with either hip BMD or lumbar BMD, after adjustment for age. However, patients with a higher BMD at the time of diagnosis had a trend toward better prognostic tumor characteristics, such as low grade, low Ki-67, PR-positive tumors, and negative lymph nodes.

Because BMD is influenced by estrogens as they inhibit bone resorption and enhance bone density (16), BMD has been suggested as a surrogate marker of lifetime estrogen exposure $(5,6)$. Numerous studies have shown an association between increased BMD and risk of breast cancer in postmenopausal women (7-13). Cauley et al. (10) found that women in the highest quartile of distal radial BMD or metacarpal bone mass had a two-to-threefold higher incidence of breast cancer when compared with women in the lowest quartile. Based on the results of these studies and others, the Breast Cancer Prevention Collaborative Group proposed BMD as a risk factor to be considered for validation and possible inclusion in future breast cancer risk prediction models (17).

Higher levels of endogenous estrogen have been identified as a risk factor of breast cancer development in postmenopausal women. Whether postmenopausal women who develop breast cancer in the setting of higher endogenous levels tend to have favorable tumor characteristics, as is observed in women who develop breast cancer in the setting of exogenous hormone use, has not been established. Zmuda et al. (8) reported that postmenopausal women with higher BMD presented 
Table 1. Patient Demographics and Baseline Clinical Characteristics by Median Lumbar and Hip BMD

\begin{tabular}{|c|c|c|c|c|c|c|c|c|}
\hline & \multicolumn{2}{|c|}{ Patient characteristics } & \multicolumn{3}{|c|}{ Lumbar BMD $\left(\mathrm{g} / \mathrm{cm}^{2}\right)$} & \multicolumn{3}{|c|}{ Hip BMD $\left(\mathrm{g} / \mathrm{cm}^{2}\right)$} \\
\hline & Frequency & Percent & $N$ & Median & p-Value* & $N$ & Median & $p$-Value* \\
\hline Total & 158 & - & 157 & 0.94 & - & 153 & 0.85 & - \\
\hline \multicolumn{9}{|l|}{ Age } \\
\hline$\leq 60$ & 63 & 39.9 & 62 & 0.95 & 0.33 & 61 & 0.87 & 0.002 \\
\hline$>60$ & 95 & 60.1 & 95 & 0.91 & & 92 & 0.81 & \\
\hline \multicolumn{9}{|l|}{ HRT } \\
\hline No & 27 & 17.1 & 27 & 0.91 & 0.70 & 27 & 0.84 & 0.87 \\
\hline Yes & 131 & 82.9 & 130 & 0.95 & & 126 & 0.85 & \\
\hline \multicolumn{9}{|c|}{ Duration of HRT } \\
\hline 0 years & 72 & 45.6 & 72 & 0.91 & 0.03 & 70 & 0.83 & 0.17 \\
\hline $0-5$ years & 23 & 14.6 & 23 & 0.91 & & 22 & 0.86 & \\
\hline$>5$ years & 63 & 39.9 & 62 & 0.98 & & 61 & 0.86 & \\
\hline \multicolumn{9}{|c|}{ Mammographic density } \\
\hline Dense & 80 & 50.6 & 79 & 0.93 & 0.79 & 77 & 0.85 & 0.97 \\
\hline Normal & 75 & 47.5 & 75 & 0.95 & & 73 & 0.86 & \\
\hline Moderate & 2 & 1.3 & 2 & 0.92 & & 2 & 0.84 & \\
\hline \multicolumn{9}{|l|}{ Histology } \\
\hline Ductal & 130 & 82.3 & 129 & 0.94 & 0.75 & 126 & 0.85 & 0.12 \\
\hline Lobular & 17 & 10.8 & 17 & 0.95 & & 16 & 0.82 & \\
\hline Other & 11 & 7.0 & 11 & 0.91 & & 11 & 0.81 & \\
\hline \multicolumn{9}{|l|}{ Tumor size } \\
\hline 1 & 120 & 76.0 & 119 & 0.93 & 0.27 & 116 & 0.85 & 0.12 \\
\hline 2 & 29 & 18.4 & 29 & 0.97 & & 28 & 0.85 & \\
\hline $3-4$ & 9 & 5.7 & 9 & 0.89 & & 9 & 0.77 & \\
\hline \multicolumn{9}{|c|}{ Positive nodes } \\
\hline No & 120 & 80.5 & 119 & 0.95 & 0.32 & 116 & 0.86 & 0.04 \\
\hline Yes & 29 & 19.5 & 29 & 0.91 & & 28 & 0.82 & \\
\hline \multicolumn{9}{|c|}{ Nuclear grade } \\
\hline 1 & 28 & 18.2 & 28 & 0.96 & 0.03 & 27 & 0.88 & 0.41 \\
\hline 2 & 97 & 63.0 & 96 & 0.96 & & 93 & 0.85 & \\
\hline 3 & 29 & 18.8 & 29 & 0.88 & & 29 & 0.81 & \\
\hline \multicolumn{9}{|l|}{ ER status } \\
\hline Negative & 4 & 2.7 & 4 & 0.88 & 0.32 & 4 & 0.85 & 0.43 \\
\hline Positive & 145 & 97.3 & 144 & 0.94 & & 140 & 0.86 & \\
\hline Missing & 9 & & 9 & & & 9 & & \\
\hline \multicolumn{9}{|l|}{ PR status } \\
\hline Negative & 14 & 9.4 & 14 & 0.89 & 0.04 & 14 & 0.84 & 0.43 \\
\hline Positive & 135 & 90.6 & 134 & 0.95 & & 130 & 0.85 & \\
\hline Missing & 9 & & 9 & & & 9 & & \\
\hline \multicolumn{9}{|l|}{ LVI } \\
\hline No & 93 & 84.6 & 92 & 0.96 & 0.01 & 89 & 0.86 & 0.02 \\
\hline Yes & 17 & 15.5 & 17 & 0.87 & & 17 & 0.81 & \\
\hline \multicolumn{9}{|l|}{$\mathrm{Ki}-67$} \\
\hline $0-17 \%$ & 50 & 70.42 & 49 & 0.97 & 0.07 & 47 & 0.85 & 0.25 \\
\hline $17-100 \%$ & 21 & 29.58 & 21 & 0.91 & & 21 & 0.82 & \\
\hline Missing & 87 & & 87 & & & 85 & & \\
\hline \multicolumn{9}{|l|}{ HER2 status } \\
\hline Negative & 137 & 95.8 & 136 & 0.95 & 0.19 & 132 & 0.85 & 0.58 \\
\hline Positive & 6 & 4.2 & 6 & 0.85 & & 6 & 0.89 & \\
\hline Missing & 15 & & 15 & & & 15 & & \\
\hline
\end{tabular}

"p-value for overall effect.

Percents may not add to $100 \%$ due to missing data.

HRT, hormone replacement therapy; ER, estrogen receptor; PR, progesterone receptor; HER2, human epidermal growth factor receptor-2; LVI, lympho-vascular invasion; BMD, bone mineral density.

with more advanced stages of disease when compared with women with lower BMD. However, in this study, we could not examine biologic tumor factors such as histologic grade and ER/PR receptor status in regard to prognosis. A recent study showed that higher lumbar spine and femoral neck BMD were associated with an increased risk of ER-positive breast cancer tumors (15).
Small numbers of HRT users in the data set lacked power to determine significant associations. Therefore, whether the trend toward more favorable tumor characteristics is due to endogenous, rather than exogenous hormone use, is difficult to determine.

In conclusion, higher BMD levels which can be used as a biomarker for higher endogenous estrogen 
Table 2. Linear Regression Models for Lumbar and Hip BMD, Adjusting for Age

\begin{tabular}{|c|c|c|c|c|}
\hline \multirow[b]{3}{*}{ Independent variables } & \multicolumn{4}{|c|}{ Dependent variable } \\
\hline & \multicolumn{2}{|c|}{ Lumbar BMD } & \multicolumn{2}{|c|}{ Hip BMD } \\
\hline & Coefficient & p-value & Coefficient & $p$-value \\
\hline \multicolumn{5}{|l|}{ HRT (Ref: No) } \\
\hline Yes & 0.02 & 0.50 & 0.02 & 0.43 \\
\hline \multicolumn{5}{|l|}{ Duration of HRT (Ref: 0 ) } \\
\hline $0-5$ years & -0.002 & 0.97 & 0.01 & 0.62 \\
\hline$>5$ years & 0.08 & 0.01 & 0.04 & 0.04 \\
\hline \multicolumn{5}{|c|}{ Mammographic density (Ref: Normal) } \\
\hline Dense & -0.01 & 0.71 & -0.01 & 0.80 \\
\hline \multicolumn{5}{|l|}{ Histology (Ref: lobular) } \\
\hline Ductal & -0.02 & 0.72 & 0.02 & 0.58 \\
\hline Other & -0.07 & 0.26 & -0.07 & 0.17 \\
\hline \multicolumn{5}{|l|}{ Tumor size (Ref: 1) } \\
\hline 2 & 0.05 & 0.12 & 0.00 & 0.99 \\
\hline $3-4$ & -0.04 & 0.47 & -0.06 & 0.17 \\
\hline \multicolumn{5}{|l|}{ Positive nodes (Ref: No) } \\
\hline Yes & -0.03 & 0.37 & -0.06 & 0.03 \\
\hline \multicolumn{5}{|l|}{ Nuclear grade (Ref: 1 ) } \\
\hline 2 & -0.02 & 0.62 & -0.03 & 0.18 \\
\hline 3 & -0.09 & 0.03 & -0.05 & 0.13 \\
\hline \multicolumn{5}{|c|}{ ER status (Ref: Negative) } \\
\hline Positive & -0.08 & 0.25 & 0.01 & 0.99 \\
\hline \multicolumn{5}{|c|}{ PR status (Ref: Negative) } \\
\hline Positive & -0.07 & 0.04 & -0.02 & 0.36 \\
\hline \multicolumn{5}{|l|}{ LVI (Ref: No) } \\
\hline Yes & -0.09 & 0.04 & -0.04 & 0.22 \\
\hline \multicolumn{5}{|l|}{ Ki - 67 (Ref: 0-17\%) } \\
\hline $17-100 \%$ & -0.09 & 0.04 & -0.05 & 0.14 \\
\hline \multicolumn{5}{|c|}{ HER2 status (Ref: Negative) } \\
\hline Positive & -0.10 & 0.17 & 0.01 & 0.83 \\
\hline
\end{tabular}

*p-value for overall effect.

HRT, hormone replacement therapy; ER, estrogen receptor; PR, progesterone receptor HER2, human epidermal growth factor receptor-2; LVI, lympho-vascular invasion; BMD, bone mineral density.

levels in postmenopausal women with breast cancer were associated with tumors with favorable prognostic features. Future studies with larger prospective cohorts are needed to validate these findings and to determine whether BMD can be incorporated in breast cancer risk prediction models.

\section{Acknowledgments}

Partially funded by NCI 2 U10 CA045809; the Lynn Cohen Breast and Ovarian Cancer Project, and the Nelly B. Connally Breast Cancer Research Fund.

\section{REFERENCES}

1. Travis RC, Key TJ. Oestrogen exposure and breast cancer risk. Breast Cancer Res 2003;5:239-47.

2. Key TJ, Pike MC. The role of oestrogens and progestagens in the epidemiology and prevention of breast cancer. Eur J Cancer Clin Oncol 1988;24:29-43.

3. Key T, Appleby P, Barnes I, Reeves G. Endogenous sex hormones and breast cancer in postmenopausal women: reanalysis of nine prospective studies. J Natl Cancer Inst 2002;94:606-16.

4. Hankinson SE, Manson JE, London SJ, Willett WC, Speizer FE. Laboratory reproducibility of endogenous hormone levels in postmenopausal women. Cancer Epidemiol Biomark Prevent 1994;3:51-6.

5. Eriksen EF, Colvard DS, Berg NJ, et al. Evidence of estrogen receptors in normal human osteoblast-like cells. Science 1988;241:84-6.

6. Turner RT, Riggs BL, Spelsberg TC. Skeletal effects of estrogen. Endocr Rev 1994;15:275-300.

7. Zhang Y, Kiel DP, Kreger BE, et al. Bone mass and the risk of breast cancer among postmenopausal women. New Eng J Med 1997;336:611-7.

8. Zmuda JM, Cauley JA, Ljung BM, Bauer DC, Cummings SR, Kuller LH. Bone mass and breast cancer risk in older women: differences by stage at diagnosis. J Natl Cancer Inst 2001;93: 930-6.

9. Buist DS, LaCroix AZ, Barlow WE, et al. Bone mineral density and endogenous hormones and risk of breast cancer in postmenopausal women (United States). Cancer Causes Control 2001;12: 213-22.

10. Cauley JA, Lucas FL, Kuller LH, Vogt MT, Browner WS, Cummings SR. Bone mineral density and risk of breast cancer in older women: the study of osteoporotic fractures. Study of Osteoporotic Fractures Research Group. JAMA 1996;276:1404-8.

11. Nguyen TV, Center JR, Eisman JA. Association between breast cancer and bone mineral density: the Dubbo Osteoporosis Epidemiology Study. Maturitas 2000;36:27-34.

12. Ganry O, Tramier B, Fardellone P, Raverdy N, Dubreuil A. High bone-mass density as a marker for breast cancer in post-menopausal women. Breast 2001;10:313-7.

13. van der Klift M, de Laet CE, Coebergh JW, Hofman A, Pols HA. Bone mineral density and the risk of breast cancer: the Rotterdam Study. Bone 2003;32:211-6.

14. Grenier D, Cooke AL, Lix L, Metge C, Lu H, Leslie WD. Bone mineral density and risk of postmenopausal breast cancer. Breast Cancer Res Treat 2011;126:679-86.

15. Singletary SE, Greene FL. Revision of breast cancer staging: the 6th edition of the TNM Classification. Semin Surg Oncol 2003;21:53-9.

16. Villareal DT, Rupich RC, Pacifici R, et al. Effect of estrogen and calcitonin on vertebral bone density and vertebral height in osteoporotic women. Osteoporos Int 1992;2:70-3.

17. Santen RJ, Boyd NF, Chlebowski RT, et al. Critical assessment of new risk factors for breast cancer: considerations for development of an improved risk prediction model. Endocr Relat Cancer 2007;14:169-87. 